\title{
Properties of electromagnetic wave propagation emerging in 2-D periodic plasma structures
}

\author{
$\operatorname{AUTHOR(S):~}$
}

Sakai, O; Tachibana, K

\section{CITATION:}

Sakai, O ... [et al]. Properties of electromagnetic wave propagation emerging in 2-D periodic plasma structures. IEEE TRANSACTIONS ON PLASMA SCIENCE 2007, 35(5): 1267 1273

\section{ISSUE DATE:}

2007-10

URL:

http://hdl.handle.net/2433/50122

\section{RIGHT:}

(c)2007 IEEE. Personal use of this material is permitted. However, permission to reprint/republish this material for advertising or promotional purposes or for creating new collective works for resale or redistribution to servers or lists, or to reuse any copyrighted component of this work in other works must be obtained from the IEEE. 


\title{
Properties of Electromagnetic Wave Propagation Emerging in 2-D Periodic Plasma Structures
}

\author{
Osamu Sakai, Member, IEEE, and Kunihide Tachibana, Member, IEEE
}

\begin{abstract}
Periodic structures in plasmas form unique dispersions of electromagnetic waves, which are examined in numerical calculation. Above electron plasma frequency, where waves equivalently propagate in dielectric media, plasma arrays with 2-D periodicity produce frequency regions of forbidden propagation, like band gaps in a photonic crystal. Below electron plasma frequency, where waves are usually forbidden to propagate due to cutoff phenomenon, bulk plasmas with periodic holes can become wave-propagating media in which localized surface modes play an important role. Such features give rise to concepts of dynamic wave controllers that change their inner electron density in time.
\end{abstract}

Index Terms-Electromagnetic propagation, microwave devices, numerical analysis, plasma devices, plasmons.

\section{INTRODUCTION}

$\mathbf{I}$ T IS A COMMON knowledge that an electromagnetic wave propagates in a nonmagnetized plasma above electron plasma frequency $\omega_{\mathrm{pe}} / 2 \pi$, which is determined by electron density $n_{\mathrm{e}}$. The equivalent dielectric constant is well described by the Drude model, and it approaches vacuum value when the wave frequency $\omega / 2 \pi$ is much higher than $\omega_{\text {pe }} / 2 \pi$. Below $\omega_{\text {pe }} / 2 \pi$, the electromagnetic wave cannot propagate into the bulk region and is reflected in a thin evanescent layer on its surface. In other words, the dielectric constant is negative in an overdense plasma, and the waves penetrate within the skin depth in general.

Such an explanation is invalid when a plasma has a finite size and/or periodic structures. One example was "plasma phonic crystal" [1], which was experimentally and theoretically verified in our previous reports [2]-[5]. It is composed of a 2-D periodic array of columnar plasmas, and electromagnetic waves well above $\omega_{\text {pe }} / 2 \pi$ may face cutoff condition in certain frequency regions; band gaps form in a similar mechanism to a photonic band gap in a usual photonic crystal composed of solid dielectric materials.

Based on this fact and its extrapolation, one can anticipate other aspects of abnormal and distinctive wave propagation in a plasma with periodic structures, which are the main targets

Manuscript received March 4, 2007; revised July 5, 2007. This work was supported in part by the Grants-in-Aid for Scientific Research from the Japanese Ministry of Education, Culture, Sports, Science, and Technology, and in part by the Industrial Technology Research Grant Program in 2006 from the New Energy and Industrial Technology Development Organization (NEDO) of Japan.

The authors are with the Department of Electronic Science and Engineering, Kyoto University, Kyoto-daigaku Katsura, Nishikyo-ku, Kyoto 615-8510, Japan (e-mail: osakai@ kuee.kyoto-u.ac.jp).

Color versions of one or more of the figures in this paper are available online at http://ieeexplore.ieee.org.

Digital Object Identifier 10.1109/TPS.2007.906133 of this paper. After reviewing band diagrams in a 2-D plasma array, we examine wave propagation in the vicinity of a band gap above $\omega_{\text {pe }} / 2 \pi$ and on flatbands below $\omega_{\text {pe }} / 2 \pi$ in the same structure. At the band gap, where wave propagation is forbidden in the frequency range above $\omega_{\mathrm{pe}} / 2 \pi$, wave field patterns show totally different features between its upper and lower sides. Flat bands with very low group velocity emerge in a certain range of frequency below $\omega_{\mathrm{pe}} / 2 \pi$, and localized surface modes contribute wave propagation along them [4] in a similar manner to localized surface plasmon in metallic photonic crystals [6]. The latter part of this paper describes wave-propagation phenomena in a bulk plasma with holes in a 2-D array pattern. In this case, we again observed band gaps above $\omega_{\text {pe }} / 2 \pi$. We also observed that wave propagation is permitted on the flatbands below $\omega_{\mathrm{pe}} / 2 \pi$; there is no continuous vacuum gap in the plasma, unlike the case of 2-D array of columnar plasmas. The properties of these waves are investigated by calculated dispersion relation and electric field profiles in comparison with the surface plasmons on the metallic surface.

\section{NumericAl Methods}

The numerical methods used here were described in our previous paper [3], [4], which will be briefly reviewed in the following.

Dielectric constant in a plasma is assumed to be in the Drude type, and $n_{\mathrm{e}}$ is fixed throughout the calculation; we assume that power carried by electromagnetic waves is so small that there is no interaction like plasma production by waves, and plasmas are maintained by another external power supply. The periodicity of the plasma structures that we will examine is restricted to a square lattice.

The dispersion relation of electromagnetic waves, or band diagram in the notation of crystal theory, is derived using two methods. A 2-D spatial periodicity of the frequency-dependent dielectric constant is taken into account. Modified plane-wave expansion method can deal with periodic structure with the dielectric constant of the Drude type [4], [7]. This method solves nonlinear eigenvalue problems with equations, including frequency $\omega / 2 \pi$ and wavenumber $k$, and the relation between $\omega$ and $k$ is analytically clarified. It is necessary to derive adequate coefficients for spatial Fourier transform in terms of a dielectric constant profile before solving the eigenvalue problem.

Another method is based on the numerical calculation of the wave equation using the finite difference method, which is referred to in this paper as the direct complex-amplitude method. Usually, one lattice cell with a unit dielectric profile is solved, and the boundary condition is set to be periodic, 


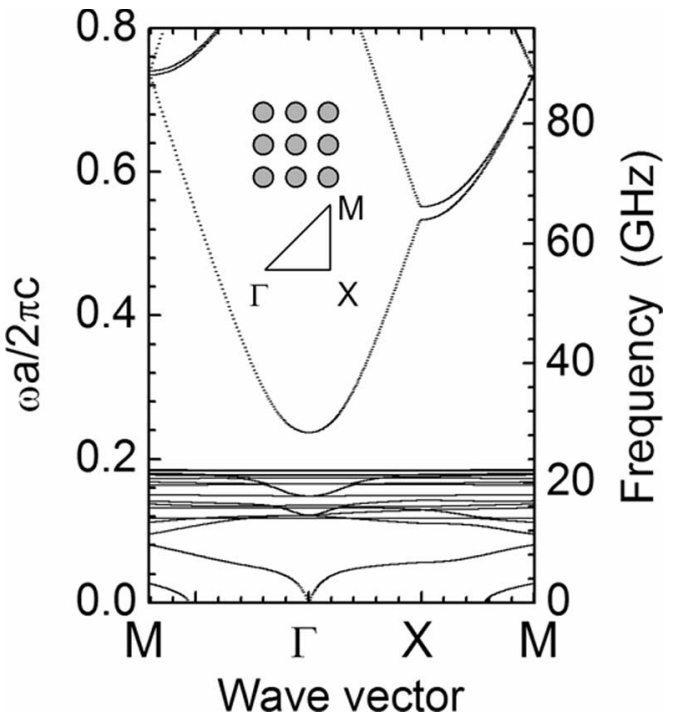

Fig. 1. Band diagram of the TE mode in square lattice of plasma columns by plane-wave expansion method. Lattice constant $a$ is $2.5 \mathrm{~mm}$. Columnar plasma with $1.75 \mathrm{~mm}$ in diameter is collisionless, and $n_{\mathrm{e}}=10^{13} \mathrm{~cm}^{-3}$.

i.e., the Bloch theorem. The field is assumed to vary with $\exp [j(\omega t-\boldsymbol{k} \cdot \boldsymbol{x})]$, where $t$ and $\boldsymbol{x}$ are the time and the position vector, respectively. That is, the numerical solution of the wave equation shows static fields with specific amplitude and phase at frequency $\omega / 2 \pi$, unlike the finite domain time difference method. A pair of $\omega$ and $k$ along the propagation branch is searched for in their 2-D plane, and arbitrary distribution of dielectric constant within one lattice cell can be assumed. Another advantage of this method is the subproducts of the field profiles in each calculation step, although the calculation for the entire band diagram requires much longer calculation time than the modified plane-wave expansion method. This method was also successfully applied to a supercell configuration to observe wave propagation around the band gap [5].

After a brief look at a band diagram derived by the modified plane-wave expansion method, in the next section, we examine the field distribution of propagating waves as well as the band diagrams using the direct complex-amplitude method. Propagation is assumed to be in the transverse-electric-field (TE) mode, where electric fields are in parallel to the 2-D periodic plane. Calculated results are all in the collisionless case with $n_{\mathrm{e}}=10^{13} \mathrm{~cm}^{-3}$. Effects of electron elastic collisions which might damp electromagnetic waves were examined in our previous report [4], and it was concluded that heavy collisionality moulds the band diagrams. However, in the case of our previous report on experimental verifications of band gaps [5], collision frequency was approximately half of the electron plasma frequency, and the band diagram in such a case varies only slightly without significant differences [4].

\section{NumericAl RESUlts AND Discussion}

\section{A. Propagation in a 2-D Plasma Array Above Electron Plasma Frequency}

Fig. 1 shows a band diagram in an infinite 2-D array of columnar plasmas, derived by the modified plane-wave expan-

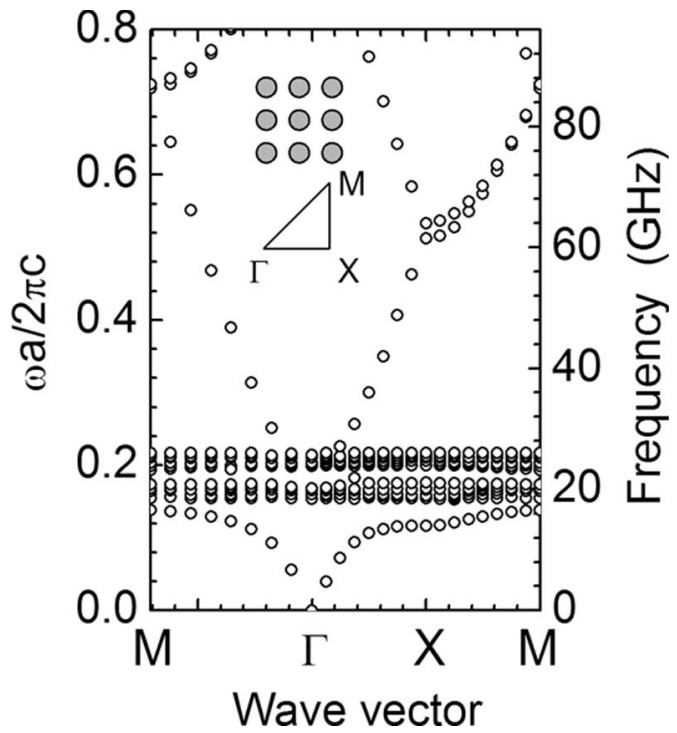

Fig. 2. Band diagram of the TE mode in square lattice of plasma columns by direct complex-amplitude method. Lattice constant $a$ is $2.5 \mathrm{~mm}$. Columnar plasma with $1.75 \mathrm{~mm}$ in diameter is collisionless, and $n_{\mathrm{e}}=10^{13} \mathrm{~cm}^{-3}$.

sion method. Here, we assumed a square lattice composed of circular plasmas with a slab $n_{\mathrm{e}}$ profile of $10^{13} \mathrm{~cm}^{-3}$ and a lattice constant of $a=2.5 \mathrm{~mm}$. The horizontal axis shows wave vector in the first Brillouin zone in a square lattice by familiar notation in usual crystal theory; points in the Brillouin zone $\Gamma$, $X$, and $M$ are wavenumber $=\pi / a$ times $[0,0],[0,1]$, and $[1,1]$, respectively. Electron plasma frequency $\omega_{\mathrm{pe}} / 2 \pi$ was around $28 \mathrm{GHz}$. The diameter of the plasma was $1.75 \mathrm{~mm}$, and therefore, the filling fraction of the plasma region inside one lattice cell was 0.38 . The first band gap in the $\Gamma-X$ direction emerged at $60-65 \mathrm{GHz}$ or $\omega a / 2 \pi c \sim 0.5$, which is a similar position to that in dielectric photonic crystals. Below $\omega_{\mathrm{pe}} / 2 \pi$, flatband region ranged from 0.37 to $0.82 \omega / \omega_{\mathrm{pe}}$. Surface wave mode or Fano mode was observed below the flatband region, which is along the vacuum propagation path and joins the lowest flatband as the frequency increases.

Fig. 2 shows a band diagram in a similar condition to Fig. 1, derived by the direct complex-amplitude method. A 2-D plane was discretized into $20 \times 20$ meshes in one square-shaped lattice cell. It clarified almost the same features shown in Fig. 1, including the band gaps, the flatbands, and the Fano mode. We also successfully obtained a case with a gradient electrondensity profile, which increases the width of the flatband region due to lower density region in the periphery [4] without changing other propagation properties. The details about the flatband regions will be described in the next section. In particular, the flatband region occupies up to $\omega_{\text {pe }} / 2 \pi$ in Fig. 2, while there is a vacant region in Fig. 1, which will be discussed using the calculated electric field profiles.

Unidirectional band gap in the $\Gamma-X$ direction, which lies around $61 \mathrm{GHz}$ in Figs. 1 and 2, is examined in the following. Forbidden propagation is enhanced due to anisotropic wave propagation in the vicinity of the band gap [1]. Here, we focus on wave field profiles around the plasma columns. Fig. 3(a) and (b) shows the electric field profiles obtained as subproducts of the band calculation shown in Fig. 2 by 
(a) $61.4 \mathrm{GHz}$

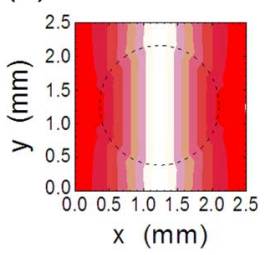

(c) $66.9 \mathrm{GHz}$

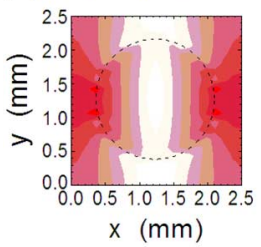

(e)

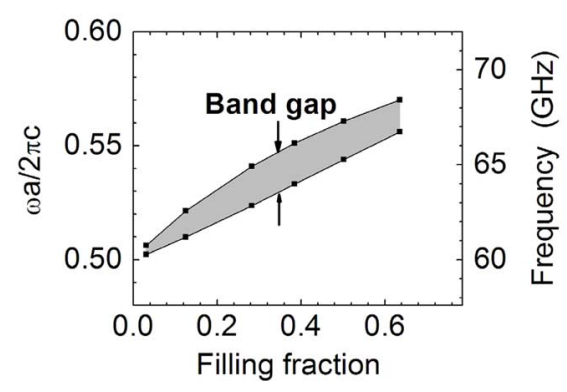

Fig. 3. Calculated profiles of electric fields normalized in amplitude in case of $k_{x} a / 2 \pi=0.50$ and $k_{y}=0$. In (a) and (b), parameters used are similar to Fig. 2. In (c) and (d), parameters used are similar to Fig. 2 except that $n_{\mathrm{e}}=$ $4 \times 10^{13} \mathrm{~cm}^{-3}$. (e) Band-gap region as a function of filling fraction of plasma area in a lattice. $n_{\mathrm{e}}$ is set to be $1 \times 10^{13} \mathrm{~cm}^{-3}$.

the direct complex-amplitude method. Electric fields greatly showed different patterns just below or above this band gap; their amplitude was smaller in the plasma region than in the outer area just below the band gap $(61.4 \mathrm{GHz})$, but their maximum region spreads over the center of the plasma just above the band gap $(64.0 \mathrm{GHz})$. These structures were similar to 1-D standing waves, and effects of the plasma with circular cross section were ambiguous.

To clarify the impacts of plasmas in the vicinity of the band gap, we calculated the electric field profiles just below or above this band gap in the case with $n_{\mathrm{e}}=4 \times 10^{13} \mathrm{~cm}^{-3}$, as shown in Fig. 3(c) and (d), which was higher than in the case in Fig. 3(a) and (b), and therefore, spatial discontinuity of dielectric constant was more distinct. Just below the band gap $(66.9 \mathrm{GHz})$, the electric field was the minimum in the center of the plasma. On the contrary, just above the band gap $(76.3 \mathrm{GHz})$, the electric field was peaking in the center of the plasma.

This fact indicates that the waves belong to completely different bands instead of the close frequency gap at the same wavenumber. Field profiles were investigated on the upper and lower sides of the band gap in solid-dielectric photonic crystals [8], [9]. In the "dielectric band" of the solid-dielectric photonic crystal, which is the first and lowest band, fields are localized in the region of the higher dielectric constant. On the contrary, in the "air band," which is the upper band of the band gap, fields are localized in the region of the lower dielectric constant. In our study, the region of the lower dielectric constant lies in the plasma region, and the field profiles in Fig. 3(a) and (b) are consistent with the results of the aforementioned solid-dielectric photonic band. This difference arises from the frequency gap that is equivalent to the difference of stored energy.

Fig. 3(e) shows the frequency range of this band gap as a function of the filling fraction of the plasma region inside one lattice cell, calculated by the modified plane-wave expansion method. The band gap shifted to higher frequency as the filling fraction increased, and the width of the band gap was at maximum when the filling fraction was 0.4. Since the spaceaveraged dielectric constant becomes lower than unity as the filling fraction increases, band gap emerges for $\omega a / 2 \pi c>0.5$. The band-gap width also becomes wider as $\omega_{\mathrm{pe}} / 2 \pi$ increases, and the dielectric constant in plasmas becomes lower at the band-gap frequency [see, for instance, Fig. 3(a)-(d)]. In general, a distinct change of the dielectric constant in space or in values widens the band gap.

The feature of wave propagation inside the band gap is evanescent in the array region, which can be analyzed in the supercell method [5]. Since this band gap shows a function of the bandstop filter, we obtain a dynamic bandstop filter by changing $n_{\mathrm{e}}$ and $a$.

\section{B. Propagation in a 2-D Plasma Array Below Electron Plasma Frequency}

As shown in Figs. 1 and 2, flatbands with very low group velocity region are present below $\omega_{\mathrm{pe}} / 2 \pi$. Such a wide frequency range arises from both localized surface modes and their periodicity.

Surface plasmon around a metal particle was well investigated in the photon frequency range [10]. When electromagnetic waves encounter an individual metal particle smaller than the wavelength, they are coupled with localized surface modes called "surface plasmon polalitons." Maximum frequency is $\omega_{\text {pe }} /\left(1+\varepsilon_{\mathrm{d}}\right)^{1 / 2}$, where $\varepsilon_{\mathrm{d}}$ is the dielectric coefficient of the medium surrounding the metal particle. The localized surface modes have azimuthal (angular) mode number $l$ around the particle, and $l$ becomes larger as the frequency approaches $\omega_{\text {pe }} /\left(1+\varepsilon_{\mathrm{d}}\right)^{1 / 2}$. In fact, as shown in Fig. 1 , there seems to be a narrow perfect photonic band gap between the lowest frequency of the equivalently dielectric branch $(\sim 28 \mathrm{GHz})$ and the highest frequency of the flatband region $(\sim 23 \mathrm{GHz})$. The highest frequency of the flatband decreases as the dielectric constant of the surrounding medium increases (not shown here), which is qualitatively consistent with change of the value of $\omega_{\mathrm{pe}} /\left(1+\varepsilon_{\mathrm{d}}\right)^{1 / 2}$.

In our case, however, structure periodicity complicates the problem. Recently, several reports about metallic photonic crystals [6], [7], [11]-[13] have dealt with this issue. We investigated the electric field profiles calculated by the direct complexamplitude method along the band branches to clarify the roles of surface plasmon and its periodic effects. Fig. 4 shows several amplitude profiles of electric fields in the propagating waves in 2-D columnar plasmas, in the same parameters as Figs. 1-3.

Electric fields in the Fano mode, present below the flatband region, are shown in Fig. 4(a). The amplitude of the electric field inside the columnar plasma was very small, and most 
(a) $12.7 \mathrm{GHz}$

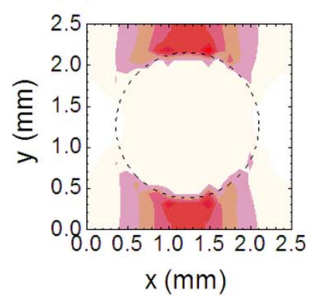

(d) $19.3 \mathrm{GHz}$

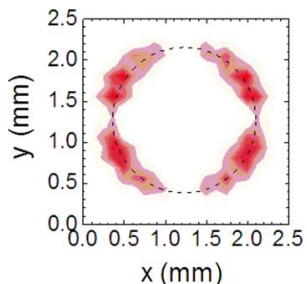

(g) $25.2 \mathrm{GHz}$

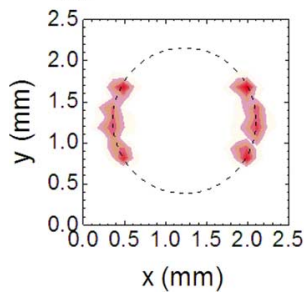

(b) $18.5 \mathrm{GHz}$

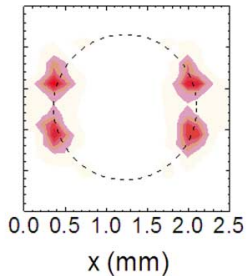

(e) $19.9 \mathrm{GHz}$

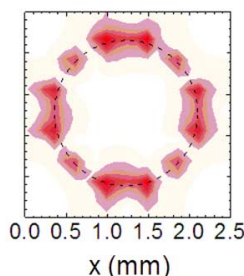

(h) $26.1 \mathrm{GHz}$

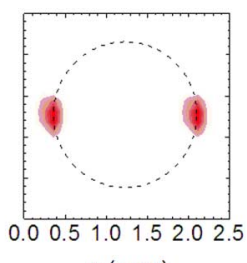

$x(\mathrm{~mm})$ (c) $19.0 \mathrm{GHz}$

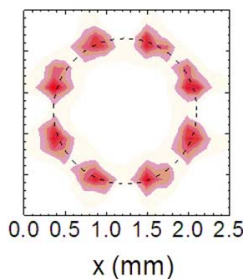

(f) $24.6 \mathrm{GHz}$

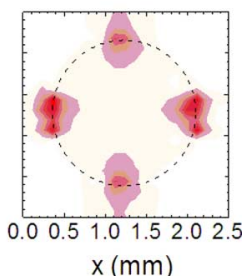

(i) $36.0 \mathrm{GHz}$

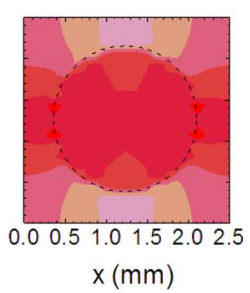

Fig. 4. Calculated profiles of electric fields normalized in amplitude in case of $k_{x} a / 2 \pi=0.25$ and $k_{y}=0$. Parameters used are similar to Fig. 2 .

of the wave energy was uniformly distributed and flowed outside the plasma. As we mentioned earlier, this wave branch coalesces with the flatbands at their lowest frequency as the frequency increases.

Electric fields of the waves on flatbands are shown in Fig. 4(b)-(h). A clearly different point from Fig. 4(a) is that electric fields were localized on the boundary between the plasma and the vacuum. Another unique feature was the change of $l$ of the standing waves around the plasma column. At lower frequency, $l$ around the plasma column was low, and it became multiple at higher frequency. This tendency is consistent with the general phenomena of surface plasmons around the metal particle. The highest $l$ number $(\sim 6)$ was observed around $20 \mathrm{GHz}$, as shown in Fig. 4(e), and this frequency was approximately in the condition of $\omega_{\mathrm{pe}} / 2^{1 / 2}$ which agrees with the predicted top frequency of the surface plasmon around the metal sphere $\left[\omega_{\text {pe }} /\left(1+\varepsilon_{\mathrm{d}}\right)^{1 / 2}\right.$ in the case where the surrounding medium is a vacuum $\left.\left(\varepsilon_{\mathrm{d}}=1\right)\right]$.

However, the sequence of $l$ along the frequency axis was not perfect as the surface plasmons around the individual metal sphere [10]. Above $20 \mathrm{GHz}$ in Figs. 2 and 4, there are some flatbands, separated from the group below $20 \mathrm{GHz}$. In this group, however, no sequential change of $l$ was found in Fig. 4. This might arise from the periodicity, as suggested by Ito and Sakoda [6]. That is, Fig. 4(f)-(h) shows a different tendency from that below $20 \mathrm{GHz}$, and these electric field profiles imply that surface wave modes are localized in the gap region of the adjacent plasma columns and no boundary condition for standing wave around the column affects them. Note that this

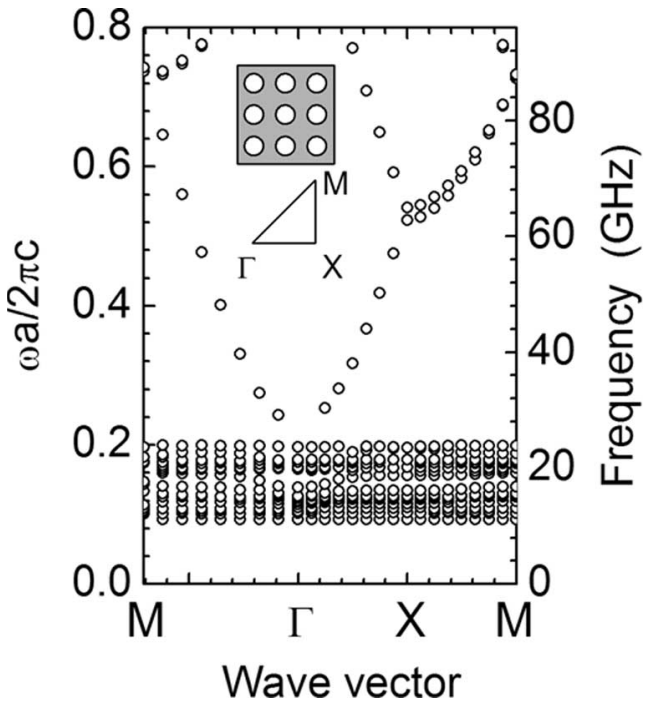

Fig. 5. Band diagram of the TE mode in square lattice of plasma holes by direct complex-amplitude method. Lattice constant $a$ is $2.5 \mathrm{~mm}$. Circular holes with $1.75 \mathrm{~mm}$ in diameter are in a collisionless infinite plasma with $n_{\mathrm{e}}=10^{13} \mathrm{~cm}^{-3}$.

group of the flatbands above $20 \mathrm{GHz}$ was hardly detected using the modified plane-wave method, as shown in Fig. 1, where the region with no detection of flatbands ranges from $20 \mathrm{GHz}$ to $\omega_{\text {pe }} / 2 \pi$. The structures of wave propagation are too fine to be detected in the modified plane-wave method, and therefore, an increase of assumed plane waves might be required to detect them in this method.

One additional comment on the effects of electron density gradient will be beneficial since density profile is not slab in usual experiments but parabolic or can be approximated by Bessel-function dependence. In this paper, we assumed a slab density profile. The effects of the density gradient on edge regions were discussed in our previous report [4] and mainly contribute to broaden the flatband region down to lower frequency, because lower density region on the outer side has a local electron plasma frequency lower than the center. Such an assumption of density gradient will be closer to experimental conditions, but it might make the discussion more complicated since effects of $l$ and those of local electron plasma frequency will overlap with each other.

In summary, wave propagation on the flatbands of a 2-D columnar plasma array is mainly attributed to the dispersion of the localized surface modes around an individual columnar plasma and is modified by periodicity in the plasma array. These phenomena analogically resemble light waveguides composed of metal nanoparticle chains [14]. The property observed in the aforementioned calculations will be applied to the dynamic waveguide of the electromagnetic waves composed of localized surface modes, since flatbands can intersect with the wave branches with various characteristic impedances.

\section{Propagation in a Bulk Plasma With Periodic Holes Below Electron Plasma Frequency}

So far, we have investigated wave propagation in an array of plasma columns. The next target is antiparallel structure, 
which is an infinite-size plasma with periodic holes. Above $\omega_{\text {pe }} / 2 \pi$, periodic dielectric constant in space will contribute to form a similar band diagram. When the frequency is low enough, since there is no continuous vacuum space in this structure, wave propagation below $\omega_{\text {pe }} / 2 \pi$ is considered difficult from the first guess of the wave-propagation theory in a bulk plasma.

A band diagram of infinite plasma with periodic holes, calculated by the direct complex-amplitude method, is shown in Fig. 5. The basic features are common to both diagrams in Figs. 2 and 5, and several different points from Fig. 2 can be found in Fig. 5. The first band-gap frequency in the $\Gamma-X$ direction was slightly higher, since the filling fraction of the plasma region in one lattice cell in Fig. 5 (0.62) was larger than that in Fig. 2 (0.38) and it reduced the synthetic dielectric constant above $\omega_{\text {pe }} / 2 \pi$. No Fano mode was present in the low frequency region since there was no continuous vacuum region. Note that wave propagation remained below $\omega_{\text {pe }} / 2 \pi$ and the flatband region expanded to lower frequencies, which are examined in the following.

Fig. 6 shows the electric field profiles in one lattice cell at various frequencies. In this case, no clear dependence of the azimuthal mode number $l$ on the frequency was found; for instance, $l=1$ at $12.3 \mathrm{GHz}, l=4$ at $12.7 \mathrm{GHz}$, and $l=2$ at $14.6 \mathrm{GHz}$. The path for the wave energy flow is limited to four points from the adjacent lattice cells through the short gap region between holes, and a plasma hole works as a wave cavity. Furthermore, conditions for standing eigenmodes along the inner surface of the hole are also required. In contrast, in the case of the columnar plasmas in Fig. 4, wave energy freely flows around the column, and therefore, wave patterns fulfill eigenmode conditions around the plasma columns and their periodicity. These facts yield differences between the cases of columnar plasmas and plasma holes.

This difference is clarified by numerical results in three sequential lattice cells, as shown in Fig. 7. Bloch condition was applied for the three lattice cells in the $x$-direction and for the one lattice cell in $y$-direction. Fig. 7 shows typical examples in the cases of columnar plasma array and hole array, where the filling fraction of the central circular region is set to be 0.38 (diameter $d=0.7 a$ ) to make the diameter and the gap of the circular areas comparable. Electric field pattern in the case of the columnar plasma array was only slightly affected by the adjacent columns [shown in Fig. 7(a)], whereas enhanced electric field was observed in the gap regions of the adjacent holes in Fig. 7(b). For the hole array, the only condition for wave energy flow is an enhanced field on the boundary by surface modes that compete against the strong decay in the plasma region.

It is difficult to express the penetration depth of the electromagnetic waves in surface plasmon in a simple formula [10], but here, for the first approximation, we estimate usual skin depth $\delta$ on the plasma surface with a slab $n_{\mathrm{e}}$ profile instead. We use the well-known definition in a collisionless plasma as $\delta=c / \omega_{\text {pe }}$, where $c$ is the velocity of light, and $\delta$ is $1.7 \mathrm{~mm}$ using the assumed $n_{\mathrm{e}}$ value in the aforementioned calculation as $10^{13} \mathrm{~cm}^{-3}$. Since this value is comparable to

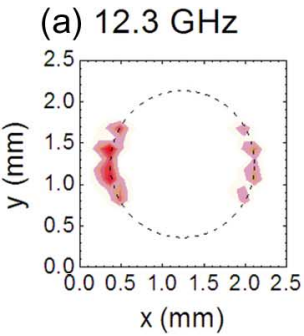

(b) $12.7 \mathrm{GHz}$
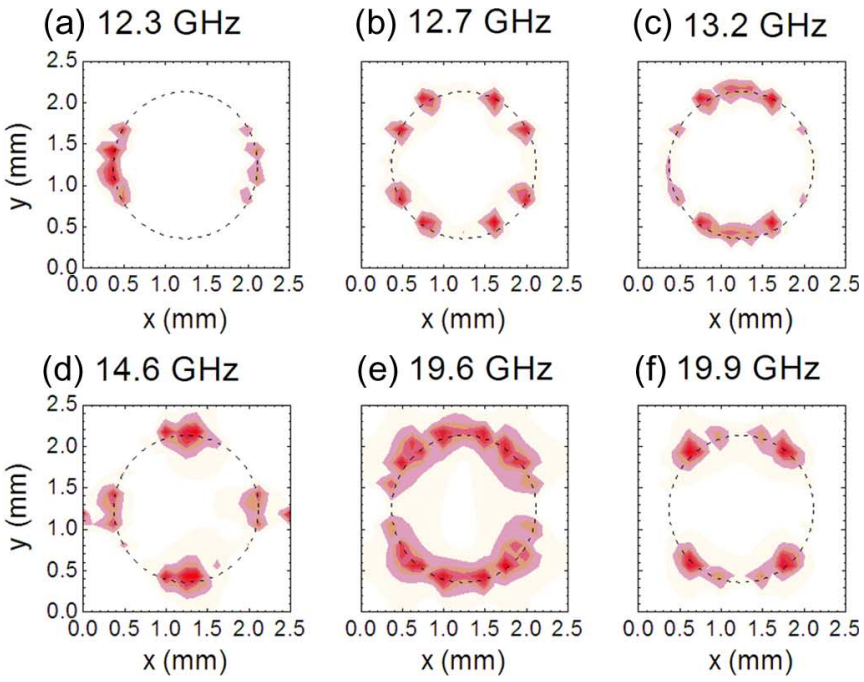

(e) $19.6 \mathrm{GHz}$
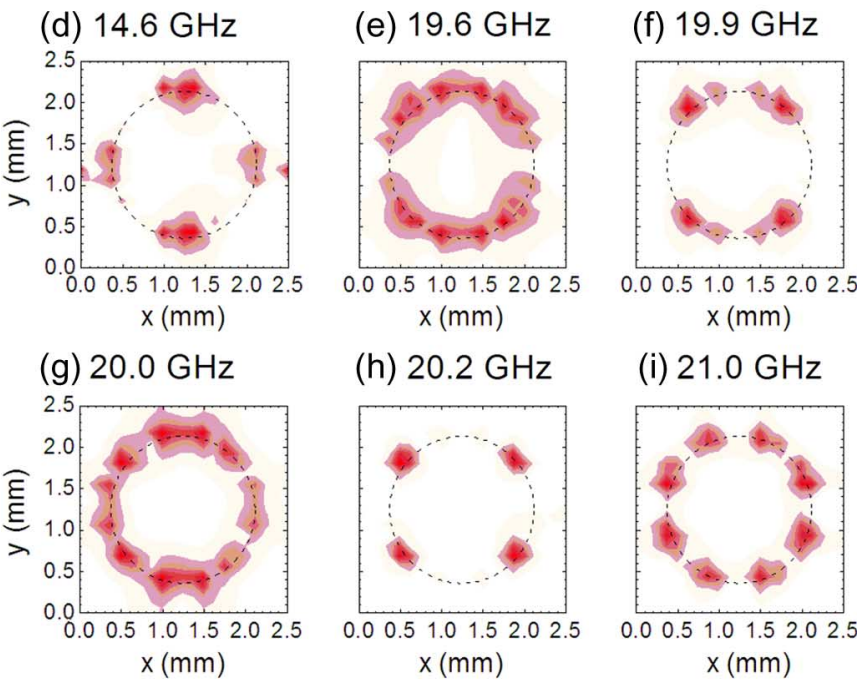

(h) $20.2 \mathrm{GHz}$

(i) $21.0 \mathrm{GHz}$
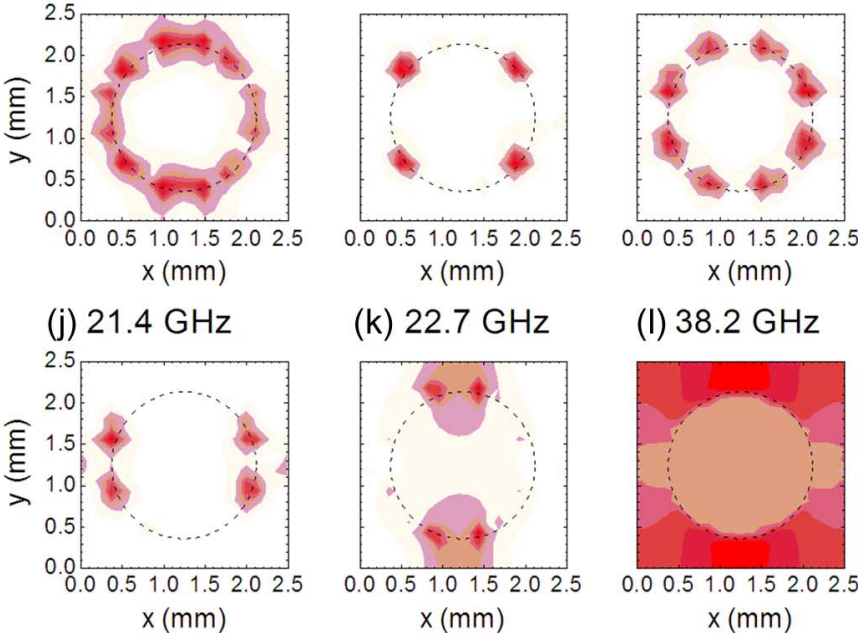

(k) $22.7 \mathrm{GHz}$

(I) $38.2 \mathrm{GHz}$
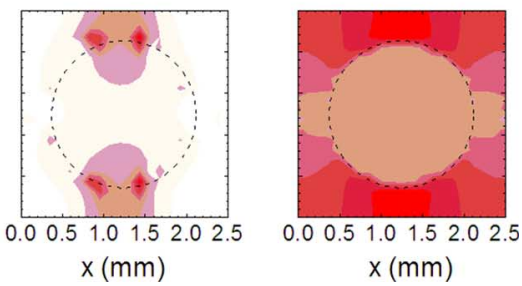

Fig. 6. Calculated profiles of electric fields normalized in amplitude in case of $k_{x} a / 2 \pi=0.25$ and $k_{y}=0$. Parameters used are similar to Fig. 5 .

the size and the gap of the plasma(s) in the aforementioned calculation, no wave propagation is expected in the normal cases in the cutoff condition. That is, wave propagation in the case of the hole array is supported not only by tunneling effects but also by resonant field enhancement on the boundary that can amplify the local fields that strongly decay in the plasma region but couple with those in adjacent cells as near fields.

These unique wave-propagation phenomena in bulk plasmas with hole array are completely different from wave transmission through a metallic plate with hole arrays. The latter is the case where waves perpendicularly propagate to the metal (and two-dimensionally periodic) plane and are basically attributed to spoof surface plasmons due to waveguide effects [15]. On the contrary, in our case shown in the numerical results, waves propagate along the two-dimensionally periodic plane. Using metals and waves in the photon range, similar phenomena will be found when holes are made in the 2-D lattice structure in the bulk metal, and waves propagate along this 2-D plane. In that case, some amount of light will pass through the metal in the usual cutoff condition; opaque material will become transparent 
(a)
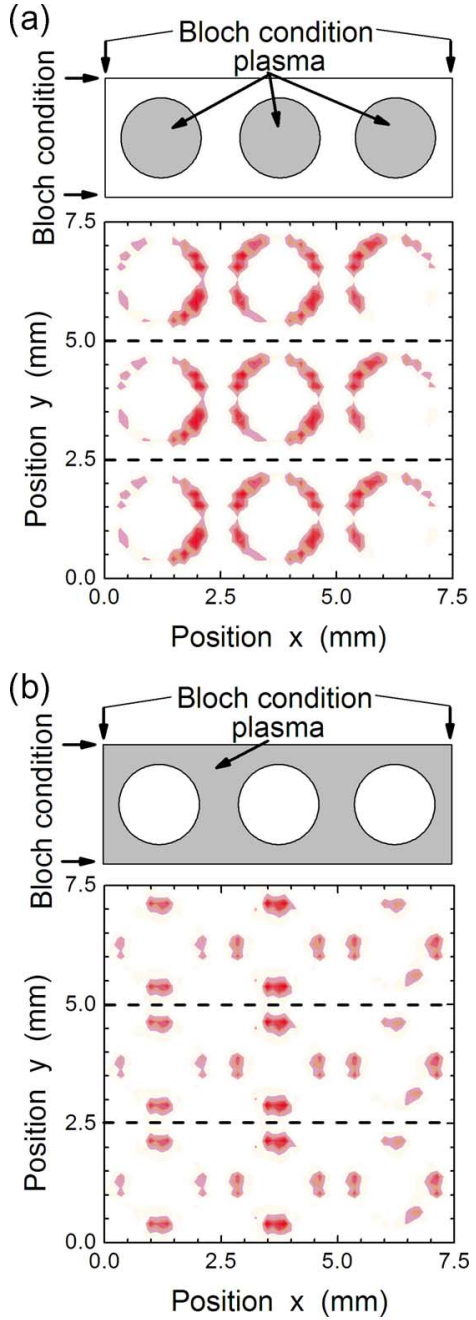

Fig. 7. Calculated profiles of electric fields in three sequential lattice cells. Upper and lower three lattice cells are repeated to see the effects from adjacent cells. (a) Plasma column array with $1.75 \mathrm{~mm}$ in diameter. $k_{x} a / 2 \pi=0.25$ and $k_{y}=0$ at $19.1 \mathrm{GHz}$. Other parameters are similar to Fig. 2. (b) Plasma hole array with $1.75 \mathrm{~mm}$ in diameter. $k_{x} a / 2 \pi=0.25$ and $k_{y}=0$ at $14.6 \mathrm{GHz}$. Other parameters are similar to Fig. 5.

to a certain extent, although damping by electron collision will be present in the actual metal material.

\section{CONCLUSION}

Using numerical methods based on the plane-wave expansion method and the finite difference method with complexamplitude fields in wave equation, we examined the distinctive wave propagation in periodic plasma structure. The band gap forms by periodic dielectric constant above the electron plasma frequency. Propagation on the flatbands below the plasma frequency in the case of a columnar plasma array is based on surface modes and their periodicity, which has similar mechanism to surface plasmons on a metal sphere. In the case of hole array in an overdense plasma, wave propagates due to the enhancement of local electric fields in surface modes. These features are unique in the microwave to terahertz region, which cannot be realized in solid media and satisfy the needs for dynamic electromagnetic wave controllers.

\section{REFERENCES}

[1] O. Sakai, T. Sakaguchi, and K. Tachibana, "Plasma photonic crystals in two-dimensional arrays of microplasmas," Contrib. Plasma Phys., vol. 47, no. 1/2, pp. 96-102, Jan. 2007.

[2] O. Sakai, T. Sakaguchi, and K. Tachibana, "Verification of a plasma photonic crystal for microwaves of millimeter wavelength range using two-dimensional array of columnar microplasmas," Appl. Phys. Lett., vol. 87, no. 24, pp. 241 505-1-241 505-3, Dec. 2005.

[3] O. Sakai, T. Sakaguchi, Y. Ito, and K. Tachibana, "Interaction and control of millimetre-waves with microplasma arrays," Plasma Phys. Control Fusion, vol. 47, no. 12B, pp. B617-B627, Dec. 2005.

[4] O. Sakai, T. Sakaguchi, and K. Tachibana, "Photonic bands in twodimensional microplasma arrays. I. Theoretical derivation of band structures of electromagnetic waves," J. Appl. Phys., vol. 101, no. 7, pp. 073 304-1-073 304-9, Apr. 2007.

[5] T. Sakaguchi, O. Sakai, and K. Tachibana, "Photonic bands in twodimensional microplasma arrays. II. Band gaps observed in millimeter and sub-terahertz ranges," J. Appl. Phys., vol. 101, no. 7, pp. 073 305-1073 305-7, Apr. 2007.

[6] T. Ito and K. Sakoda, "Photonic bands of metallic systems. II. Features of surface plasmon polaritons," Phys. Rev. B, Condens. Matter, vol. 64, no. 4, pp. $045117-1-045117-8$, Jul. 2001.

[7] V. Kuzmiak and A. A. Maradudin, "Photonic band structures of oneand two-dimensional periodic systems with metallic components in the presence of dissipation," Phys. Rev. B, Condens. Matter, vol. 55, no. 12, pp. 7427-7444, Mar. 1997.

[8] M. D. B. Charlton, M. E. Zoorob, G. J. Parker, M. C. Netti, J. J. Baumberg, S. J. Cox, and H. Kemhadjian, "Experimental investigation of photonic crystal waveguide devices and line-defect waveguide bends," Mater. Sci. Eng. B, vol. 74, no. 1, pp. 17-24, May 2000.

[9] G. Bottger, C. Liguda, M. Schmidt, and M. Eich, "Improved transmission characteristics of moderate refractive index contrast photonic crystal slabs," Appl. Phys. Lett., vol. 81, no. 14, pp. 2517-2519, Sep. 2002.

[10] F. Forstmann and R. R. Gerhardts, Metal Optics Near the Plasma Frequency. Berlin, Germany: Springer-Verlag, 1986.

[11] E. Moreno, D. Erni, and C. Hafner, "Band structure computations of metallic photonic crystals with the multiple multipole method," Phys. Rev. B, Condens. Matter, vol. 65, no. 15, pp. 155 120-1-155 120-10, Apr. 2002

[12] O. Toader and S. John, "Photonic band gap enhancement in frequencydependent dielectrics," Phys. Rev. E, Stat. Phys. Plasmas Fluids Relat. Interdiscip. Top., vol. 70, no. 4, pp. 046 605-1-046605-15, Oct. 2004.

[13] R. L. Chern, C. C. Chang, and C. C. Chang, "Analysis of surface plasmon modes and band structures for plasmonic crystals in one and two dimensions," Phys. Rev. E, Stat. Phys. Plasmas Fluids Relat. Interdiscip. Top., vol. 73, no. 3, pp. 036 605-1-036 605-15, Mar. 2006.

[14] S. A. Maier, M. L. Brongersma, P. G. Kik, and H. A. Atwater, "Observation of near-field coupling in metal nanoparticle chains using far-field polarization spectroscopy," Phys. Rev. B, Condens. Matter, vol. 65, no. 19, pp. 193 408-1-193 408-4, May 2002.

[15] J. B. Pendry, L. Martin-Moreno, and F. J. Garcia-Vidal, "Mimicking surface plasmons with structured surfaces," Science, vol. 305, no. 5685, pp. 847-848, Aug. 2004.

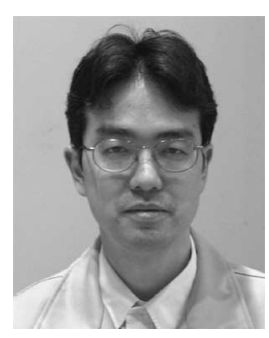

Osamu Sakai (M'05) received the B.S., M.S., and Ph.D. degrees from Kyoto University, Kyoto, Japan, in 1990, 1992, and 1996, respectively. His major field of study in the Ph.D. degree was plasma heating and confinement using electromagnetic waves in mirror plasmas.

He was a Research Engineer with the Sharp Corporation, Nara, Japan, and he is currently an Assistant Professor (Lecturer) with the Department of Electronic Science and Engineering, Kyoto University. His current research interests include microplasmas and their application to electromagnetic wave controllers.

Dr. Sakai is a member of the Japan Society of Applied Physics and the Japan Society of Plasma Science and Nuclear Fusion Research. 


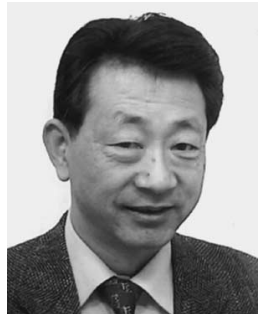

Kunihide Tachibana (M'02) received the B.S., M.S., and Ph.D. degrees from Kyoto University, Kyoto, Japan, in 1968, 1970, and 1973, respectively. He was with the Department of Electronics, Kyoto Institute of Technology, in 1974, and became a Full Professor in 1988. He moved to the Department of Electronic Science and Engineering, Kyoto University as a Full Professor in 1995. He has been the Vice-Dean of the Graduate School of Engineering, Kyoto University since 2006. His research subjects include plasma diagnostics with lasers and other spectroscopic techniques in various kinds of plasmas for material processing, light sources, and display devices. His current research interest is shifting toward microplasmas and atmospheric pressure discharges.

Dr. Tachibana is a member of the Japan Society of Applied Physics, the American Vacuum Society, etc. He serves as a Leader of the Priority Area on Microplasmas supported by the Japanese Ministry of Education, Science, Culture, and Sports. He is also the Chairman of the 153rd Committee of the Japan Society for the Promotion of Science on Plasma Material Science. 